\title{
Chemical composition and antibacterial activities of Rhus tripartita essential oils from Algeria
}

\author{
KHAOULA BENLEMBAREK ${ }^{1,}$ TAKIA LOGRADA ${ }^{1}$, MESSAOUD RAMDANI ${ }^{1, \bullet}$, GILLES FIGUEREDO ${ }^{2}$, \\ PIERRE CHALARD ${ }^{3}$ \\ ${ }^{1}$ Laboratory of Natural Resource Valorization, Faculty of Life and Natural Sciences, Ferhat Abbas University Setif-1. 19000 Setif, Algeria. \\ Tel.: +21-3658 101010, "email: ramdanimessaoud@yahoo.com, ramdanimessaoud@univ-setif.dz \\ ${ }^{2}$ LEXVA Analytique. 460 Rue Du Montant, 63110 Beaumont, France \\ ${ }^{3}$ Université Clermont Auvergne, CNRS, SIGMA Clermont, ICCF, F-63000 Clermont-Ferrand, France
}

Manuscript received: 10 November 2020. Revision accepted: 30 December 2020.

\begin{abstract}
Benlembarek K, Lograda T, Ramdani M, Figueredo G, Chalard P. 2021. Chemical composition and antibacterial activities of Rhus tripartita essential oils from Algeria. Biodiversitas 22: 480-490. The yield of Rhus tripartita essential oils in Algeria is low with an average of $0.16 \pm 0.017$. The chemical composition of the oils was determined by GC-GC / MS analysis. Seventy-eight compounds were identified with an average of $92.3 \pm 26.4 \%$ in the total oil. The $\alpha$-pinene $(58.72 \pm 14.45 \%), \Delta 3$-carene $(5.21 \pm 6.89 \%)$, caryophyllene-Z $(4.55 \pm 4.68 \%)$ and $\beta$-pinene $(3.07 \pm 185 \%)$ are the major components of the oils of $R$. tripartita populations. The results reveal a notable difference between the chemical composition of essential oils from male and female populations. The oils of this species are composed of four chemotypes, the $\Delta 3$-carene, $\alpha$-pinene; limonene chemotype characterizes the male populations of Tamanrasset and Boussaada. The $\alpha$-pinene - $\Delta 3$-carene - caryophyllene-Z chemotype characterizes the male populations of M'sila and Mergueb. The female M'sila population is characterized by the $\alpha$-pinene - $\Delta 3$-carene - $\Delta$-cadinene chemotype. The remaining populations show the $\alpha$ pinene - $\beta$-pinene - caryophyllene- $Z$ chemotype. The evaluation of antibacterial activities reveals that Staphylococcus aureus, Escherichia coli, Proteus mirabilis and Pseudomonas aeruginosa are not sensitive to the essential oils of $R$. tripartita. In contrast, Enterococcus faecalis is the most sensitive to the oils.
\end{abstract}

Keywords: Algeria, essential oils, chemotypes, antibacterial activities, Rhus tripartita

\section{INTRODUCTION}

The Rhus (Anacardiaceae) consists of approximately 200 species distributed in different temperate zones (Giovanelli et al. 2017). In Algeria, the genus Rhus is represented by three species, Rhus tripartite, $R$. coriaria, and $R$. pentaphylla (Quézel and Santa 1962). The Rhus tripartita or Searsia tripartita (Ucria) Moffett is located in arid and semi-arid North Africa (Quézel and Santa 1962) and in the steppes of Western Asia (Idm'hand et al. 2019).

Rhus tripartita is widely used in traditional and modern medicine (Lee et al. 2010; Wu et al. 2013). It is used in southern Algeria to treat diabetes, bloating, fever, kidney disease, skin lesions, diarrhea and several gut diseases (Hadjadj et al. 2015). The fruits of Rhus tripartita are eaten fresh or mixed with water, honey, or milk; the leaves are also used as animal feed; the barks and roots are used in crafts to dye leather, wool and silk (Floch 1983; Ben Mahmoud et al. 2015). The products of secondary metabolism of $R$. tripartita, in particular the phenolic compounds, have anticarcinogenic, anti-thrombotic and anti-inflammatory (Chung et al. 2010; Ben Barka et al. 2019), anti-nephritic, antimicrobial (Abbassi et al. 2012) activities; antioxidants and preventive properties of breast cancer (Wang et al. 2005; Amin et al. 2007; Shahat et al. 2016).

The chemical composition of the genus Rhus is not much studied. The essential oils of $R$. coriaria from Sicily present cembrene and $\beta$-caryophyllene as the majority products of the leaves, while the flowers are characterized by the presence of $\alpha$-pinene and tridecanoic acid (Ridel et al. 2017); the same results were obtained in Iran by Zhaleh et al. (2018). In a similar study in Turkey, the chemical profile of $R$. coriaria populations showed that the main components were $\alpha$-pinene, $\beta$-caryophyllene and cembrene (Brunke et al. 1993). Palestinian, Jordanian, and Egyptian sumac $(R$. coriaria $)$ are characterized by $\alpha$-pinene, naphthalene and cymene-O, respectively (Farag et al. 2018). Work on the chemical composition of the leaves of R. typhina has shown that monoterpenes are in the majority (Bestmann et al. 1988). The analysis of essential oils from the leaves and flowers of $R$. mysorensis allowed the estimation of the abundance of $\alpha$-pinene, limonene, sabinene and $\beta$-eudesmol (Srivastava et al. 2005).

The ethanolic extracts, from the aerial parts of $R$. tripartita from the Bechar region (Algeria), showed a weak antibacterial activity against Staphylococcus aureus, Escherichia coli, Enterobacter cloacae and Pseudomonas aeruginosa (Bereksi et al. 2018). While the chloroform extracts from Tunisian populations presented a strong antibacterial activity against $S$. aureus and strong antifungal activity against the yeast Candida albicans (Abbasi and Hani 2011).

The $R$. tripartita leaves in Libya showed, that both ethanolic extracts and chloroformic solvents, have a low inhibitory effect against $E$. coli and $P$. aeruginosa (Habibi et al. 2015). On the other hand, the studies by Tlili et al. 
(2019) show significant activity of leaf extracts against Listeria monocytogenes, S. epidermidis, and $S$. aureus (MRS); but they did not show any significant activity against Gram-negative bacteria. The hydro-methanolic extract of stems, roots and leaves of $R$. tripartita showed strong antimicrobial activity against $L$. monocytogene and Bacillus cereus (Benbarka et al. 2019).

The evaluation of the antibacterial activity of $R$. tripartita alcoholic extracts in Egypt showed significant antibacterial activity against Staphylococcus aureus and $S$. aureus (MRSA) (Abd El-Salam 2015). A similar study by Njoroge and Opiyo (2019) in Kenya shows that compounds from the root bark of $R$. natalensis exhibited moderate antibacterial and antifungal activity against Staphylococcus aureus, Bacillus subtilis, Escherichia coli, Pseudomonas aeruginosa, Candida albicans, Penicillium notatum, and Aspergillus niger. Zhaleh et al. (2018), by studying the antibacterial activity of essential oils of Rhus coriaria in Iran, showed that this species could be used as an antibiotic to treat bacterial infections.

The aim of this study is to analyze the essential oils of Rhus tripartita populations in Algeria, to compare the chemical composition of essential oils of populations, identify the chemotypes of this species and to study the antibacterial activities of different populations.

\section{MATERIALS AND METHODS}

\section{Plant materials}

The Rhus tripartita is an easily recognizable shrub due to its thorny branches and its small leaves, cut into three folioles. It is a dioecious plant with small clusters of yellowish-white flowers. The fruit is a small drupe $3-5 \mathrm{~mm}$ in diameter with a single seed inside having a spherical shape and a red to black color (Quézel and Santa 1962; Furth 1985).

The aerial parts of $R$. tripartita were collected in the flowering stage from seven localities in East Algeria (Figure 1) in February and March 2020. The species $R$. tripartita is dioecious; from each station, both male and female populations were sampled. Geographic coordinates of the stations are listed in Table 1.

Table 1. Geographical coordinates of sampled stations

\begin{tabular}{|c|c|c|c|c|c|}
\hline \multicolumn{2}{|c|}{ Localities } & Populations & $\begin{array}{c}\text { Long. } \\
\text { E }\end{array}$ & $\begin{array}{c}\text { Lat. } \\
\text { N }\end{array}$ & $\begin{array}{r}\text { Alt. } \\
\text { (m) }\end{array}$ \\
\hline \multirow[t]{8}{*}{ M'sila } & \multirow[t]{2}{*}{1.} & M'sila F & $4^{\circ} 35^{\prime} 04^{\prime \prime}$ & $35^{\circ} 49^{\prime}$ & 622 \\
\hline & & M'sila M & $4^{\circ} 35^{\prime} 04^{\prime \prime}$ & $35^{\circ} 49^{\prime}$ & 619 \\
\hline & \multirow[t]{2}{*}{2.} & Mergueb F & $3^{\circ} 57^{\prime} 22^{\prime \prime}$ & $35^{\circ} 35^{\prime} 51^{\prime \prime}$ & 630 \\
\hline & & Mergueb M & $3^{\circ} 57^{\prime} 22^{\prime \prime}$ & $35^{\circ} 35^{\prime} 51^{\prime \prime}$ & 628 \\
\hline & \multirow[t]{2}{*}{3 . } & Maïtar F & $4^{\circ} 06^{\prime} 42^{\prime \prime}$ & $35^{\circ} 14^{\prime} 11 "$ & 610 \\
\hline & & Maïtar M & $4^{\circ} 06^{\prime} 42^{\prime \prime}$ & $35^{\circ} 14^{\prime} 11 "$ & 604 \\
\hline & \multirow[t]{2}{*}{4.} & Boussaada F & $4^{\circ} 09^{\prime} 52^{\prime \prime}$ & $35^{\circ} 11^{\prime} 37^{\prime \prime}$ & 596 \\
\hline & & Boussaada M & $4^{\circ} 09^{\prime} 52^{\prime \prime}$ & $35^{\circ} 11^{\prime} 37^{\prime \prime}$ & 590 \\
\hline \multirow[t]{3}{*}{ Biskra } & 5. & Ksaiate M & $4^{\circ} 42^{\prime} 08^{\prime \prime}$ & $34^{\circ} 49^{\prime} 18^{\prime \prime}$ & 784 \\
\hline & \multirow[t]{2}{*}{6.} & Chaïba F & $4^{\circ} 56^{\prime} 38^{\prime \prime}$ & $34^{\circ} 49^{\prime} 39^{\prime \prime}$ & 417 \\
\hline & & Chaïba M & $4^{\circ} 56^{\prime} 38^{\prime \prime}$ & $34^{\circ} 49^{\prime} 39^{\prime \prime}$ & 416 \\
\hline Tamanı & 7. & Tamanrasset & $5^{\circ} 28^{\prime} 98^{\prime \prime}$ & $24^{\circ} 24^{\prime} 17^{\prime \prime}$ & 1140 \\
\hline
\end{tabular}

Note: $\mathrm{F}=$ female population; $\mathrm{M}=$ male population

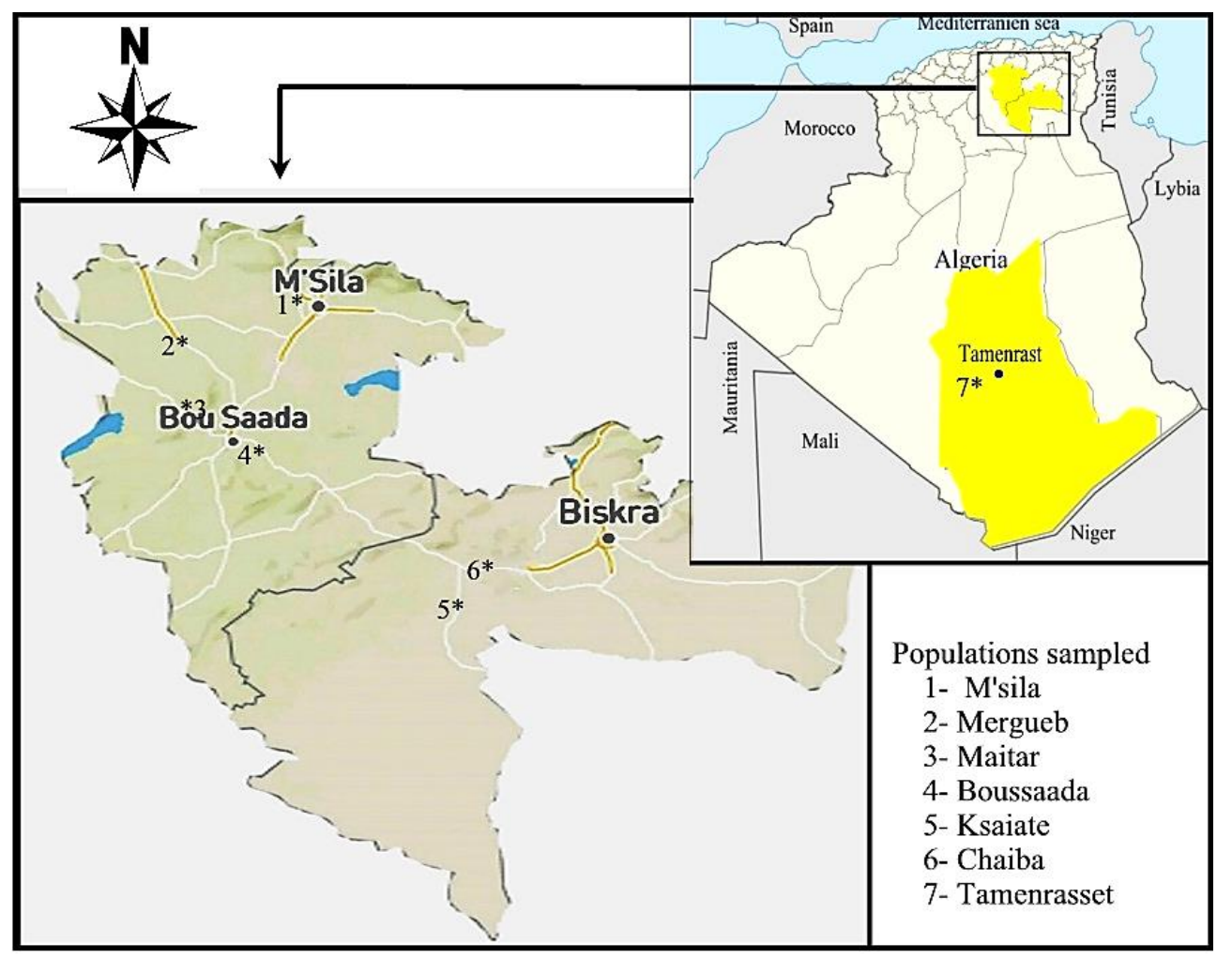

Figure 1. Populations of Rhus tripartita sampled in Algeria 


\section{The essential oils extraction}

The air-dried materials were subjected to hydrodistillation for $3 \mathrm{~h}$ using a Clevenger apparatus type. Voucher specimens were deposited in the herbarium of the Department of Biology and Ecology, Setif-1 University, Algeria (Identification of samples was assured by botanist from the Research Institute). The oil obtained was collected and dried over anhydrous sodium sulfate, and stored in screw-capped glass vials in a refrigerator at $4-5^{\circ} \mathrm{C}$ prior to analysis. The Essential oils yield was calculated by the following formula:

$$
\text { Yield }=\frac{\text { Mass of essential oil }}{\text { Mass of plant matter }} x 100
$$

\section{Essential oils analysis}

The essential oils were analyzed using a HewlettPackard gas chromatograph (CG/FID 7890), coupled to a gas chromatograph (CG/MS 7890/5975C), equipped with a Column Apolar: DB5 MS: $40 \mathrm{~m} 0.18 \mathrm{~mm} 0.18 \mu \mathrm{m}$ and programming from $50^{\circ} \mathrm{C}$ for $5 \mathrm{~min}$ at rate of $5^{\circ} \mathrm{C} / \mathrm{min}$ up to $300^{\circ} \mathrm{C}$. Helium was used as the carrier gas $(1.0 \mathrm{ml} / \mathrm{min})$; injected in the split mode (1:30), injector and detector temperature of $280^{\circ} \mathrm{C}$ with split $1 / 100$. The mass spectrometer worked in the $\mathrm{EI}$ mode at $70 \mathrm{eV}$; electron multiplier, $2500 \mathrm{~V}$; ion source temperature, $180^{\circ} \mathrm{C}$; the $\mathrm{MS}$ data were acquired in the scan mode in the $\mathrm{m} / \mathrm{z}$ range of 33450. The identification of the components was based on the comparison of their mass spectra with those of NIST mass spectral library (Masada 1976; NIST 2002) and those described by Adams, and the comparison of their retention indices either with those of authentic compounds or with literature values (Adams 2007).

\section{Antibacterial activity assessment}

The in-vitro evaluation of antimicrobial activity of Rhus tripartita essential oils was performed by the disc diffusion method, against three Gram-negative bacteria (Escherichia coli ATCC 25922, Proteus mirabilis ATCC 35659 and Pseudomonas aeruginosa ATCC 27853); and two Grampositive bacteria (Staphylococcus aureus ATCC 25923, and Enterococcus faecalis ATCC 51299). These bacteria were obtained from the Pasteur Institute of Algiers and M'sila, the applied microbiology laboratory of Setif University and Setif Hospital. The bacterial inoculums were prepared from overnight broth culture in physiological saline $(0.8 \% \mathrm{NaCl})$ to obtain an optical density ranged from 0.08 to 0.1 at $625 \mathrm{~nm}$. Muller Hinton agar (MHA) was poured in Petri dishes, solidified, and surface dried before bacteria inoculation. Sterile discs (6 $\mathrm{mm}$ ) were placed on inoculated MHA, filled with $10 \mu \mathrm{l}$ of stock solution of essential oils or diluted essential oils (1/1, $1 / 2$ and 1/3 v:v of DMSO). DMSO was used as a negative control and the antibiotics Colistin, Cefotaxime, Gentamicin and Imipenem were used as positive control. The bacterial growth inhibition was determined as the diameter of the inhibition zones around the discs. The Petri dishes were incubated at $37^{\circ} \mathrm{C}$ for 18 to $24 \mathrm{~h}$ aerobically. All the tests were performed in triplicate, and the means were calculated as results. The sensitivity to essential oils was classified by diameter of inhibition halos as follows: not sensitive (-) for diameter less than $8 \mathrm{~mm}$; sensitive (+) for diameter 9-14 mm; very sensitive $(++)$ for diameter 15 $19 \mathrm{~mm}$ and extremely sensitive $(+++)$ for diameter larger than $20 \mathrm{~mm}$ (Ponce et al. 2003).

\section{Statistical analysis}

The data were first, subjected to the Principal Components Analysis (PCA) to examine the relationships among the terpenes compounds and identify the possible structure of the populations. Cluster analysis (UPGMA) was carried out on the original variables and on the Manhattan distance matrix to seek hierarchical associations among the populations. Cluster analyzes were performed using Statistica v10 software. The data of antibacterial activity were expressed as mean \pm SD. The significant results were analyzed by the three-way ANOVA and posthoc tests using a CoStat $\mathrm{v} 6.4$ software package. Differences with $\mathrm{P}<0.05$ were considered significant.

\section{Desirability test}

A desirability test is a tool for evaluating the effect of factors or variables, which can predict the effect of each factor of the design. The scales range from zero (most unfavorable/undesirable values) to one (most desirable values).

\section{RESULTS AND DISCUSSION}

The essential oil, of Rhus tripartita, isolated by hydrodistillation from the aerial parts, were obtained in low yield $(0.16 \pm 0.02 \%)(\mathrm{v} / \mathrm{w})$. This yield is important in the altitudes that range between $596 \mathrm{~m}$ and $628 \mathrm{~m}$, for against its value decreases in the lower and higher altitudes (Figure 2). The regression curve shows a small increase in efficiency from low altitudes to high altitudes.

The composition of the chemical profile of Algerian populations of the Rhus tripartita is obtained by gas chromatography/mass spectrometry (GC-MS). The analysis of the chemical composition of essential oils, allowed us to identify seventy-eight components with an average of $92.31 \pm 26.36 \%$ of the total oil. The compounds, identified in this oil and their relative abundance, are presented in their order of appearance (Table 2).

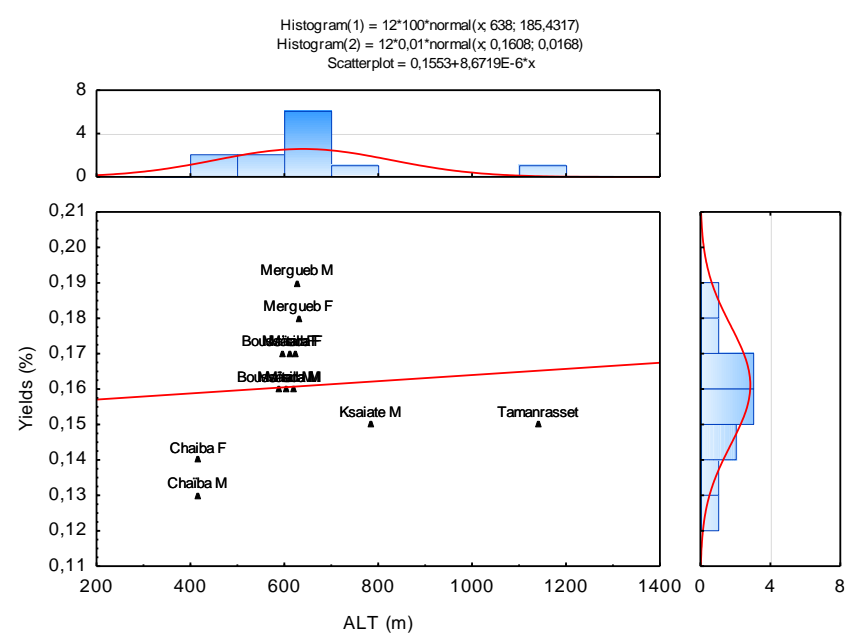

Figure 2. Yields in essential oils of Rhus tripartita 
Table 2. Chemical composition of Rhus tripartita essential oils in Algeria

\begin{tabular}{|c|c|c|c|c|c|c|c|c|c|c|c|c|c|c|c|}
\hline \multirow{2}{*}{$\begin{array}{l}\text { Stations } \\
\text { Populations }\end{array}$} & \multirow[b]{2}{*}{ IK } & \multirow{2}{*}{ 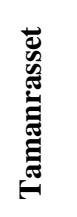 } & \multicolumn{2}{|c|}{$\begin{array}{l}\stackrel{\pi}{\bar{n}} \\
\dot{\Sigma}\end{array}$} & \multicolumn{2}{|c|}{ 这 } & \multicolumn{2}{|c|}{ 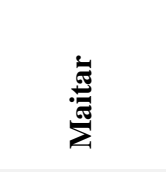 } & \multirow{2}{*}{ 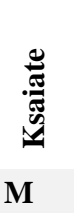 } & \multicolumn{2}{|c|}{ 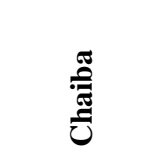 } & \multicolumn{2}{|c|}{ 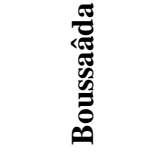 } & \multirow{2}{*}{ 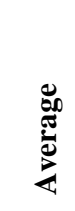 } & \multirow{2}{*}{ \% } \\
\hline & & & $\mathbf{M}$ & $\mathbf{F}$ & M & $\mathbf{F}$ & $\mathbf{M}$ & $\mathbf{F}$ & & M & $\mathbf{F}$ & M & $\mathbf{F}$ & & \\
\hline $\mathrm{Nb}$ of components & & 32 & 54 & 52 & 41 & 42 & 47 & 47 & 63 & 43 & 47 & 53 & 53 & 48 & 8 \\
\hline Yields & & 0.15 & 0.16 & 0.17 & 0.19 & 0.18 & 0.16 & 0.17 & 0.15 & 0.13 & 0.14 & 0.16 & 0.17 & 0.16 & 0.02 \\
\hline Total \% & & 96.8 & 99.6 & 97.9 & 99.9 & 99.9 & 99.9 & 99.5 & 99.5 & 99.8 & 99.9 & 99.4 & 99.8 & 92.3 & 26.4 \\
\hline Tricyclene & 921 & 0.0 & 0.0 & 0.0 & 0.0 & 0.1 & 0.0 & 0.1 & 0.1 & 0.2 & 0.0 & 0.0 & 0.1 & 0.1 & 0.1 \\
\hline$\alpha$-thujene & 927 & 0.0 & 0.0 & 0.0 & 0.0 & 0.0 & 0.0 & 0.0 & 0.1 & 0.1 & 0.4 & 0.0 & 0.0 & 0.1 & 0.1 \\
\hline$\alpha$-pinene & 934 & 35.8 & 34.6 & 54.8 & 43.5 & 64.4 & 65.6 & 69.7 & 67.6 & 66.4 & 78.4 & 52.0 & 71.9 & 58.7 & 14.5 \\
\hline$\alpha$-fenchene & 950 & 0.0 & 0.1 & 0.1 & 0.0 & 0.1 & 0.0 & 0.0 & 0.0 & 0.1 & 0.0 & 0.0 & 0.0 & 0.0 & 0.0 \\
\hline Camphene & 953 & 0.8 & 0.8 & 0.7 & 1.1 & 1.6 & 0.4 & 1.8 & 0.8 & 1.7 & 0.5 & 0.3 & 1.1 & 1.0 & 0.5 \\
\hline Sabinene & 973 & 0.0 & 0.0 & 0.1 & 0.3 & 0.0 & 0.0 & 0.0 & 0.1 & 0.0 & 0.1 & 0.0 & 0.1 & 0.1 & 0.1 \\
\hline$\beta$-pinene & 980 & 2.5 & 1.3 & 1.6 & 3.0 & 4.0 & 0.7 & 6.4 & 3.4 & 6.4 & 1.8 & 1.3 & 4.6 & 3.1 & 1.9 \\
\hline Myrcene & 989 & 2.4 & 0.5 & 1.5 & 7.7 & 5.3 & 0.7 & 1.0 & 2.4 & 2.7 & 2.8 & 3.3 & 2.1 & 2.7 & 2.1 \\
\hline$\Delta 2$-carene & 999 & 0.0 & 0.0 & 0.1 & 0.0 & 0.0 & 0.1 & 0.0 & 0.0 & 0.0 & 0.0 & 0.1 & 0.0 & 0.0 & 0.0 \\
\hline$\alpha$-phellandrene & 1008 & 0.0 & 0.0 & 0.0 & 0.0 & 0.0 & 0.0 & 0.0 & 0.3 & 0.2 & 0.2 & 0.2 & 0.1 & 0.1 & 0.1 \\
\hline$\Delta 3$-carene & 1009 & 22.3 & 6.2 & 8.3 & 7.1 & 0.4 & 0.0 & 0.0 & 0.4 & 0.0 & 0.0 & 15.3 & 2.4 & 5.2 & 7.2 \\
\hline$\alpha$-terpinene & 1017 & 1.5 & 0.1 & 0.2 & 0.2 & 0.1 & 0.1 & 0.0 & 0.3 & 0.6 & 0.4 & 1.0 & 0.1 & 0.4 & 0.4 \\
\hline Cymene-ortho & 1026 & 0.6 & 0.3 & 0.3 & 0.6 & 0.5 & 0.5 & 0.3 & 0.5 & 0.6 & 2.1 & 0.4 & 0.2 & 0.5 & 0.5 \\
\hline Limonene & 1031 & 7.2 & 2.2 & 2.1 & 2.3 & 1.3 & 0.8 & 1.1 & 1.6 & 2.3 & 2.0 & 6.0 & 2.2 & 2.6 & 2.0 \\
\hline$\beta$-phellandrene & 1032 & 0.4 & 0.2 & 0.2 & 0.3 & 0.3 & 0.3 & 0.3 & 0.6 & 0.6 & 0.5 & 0.8 & 0.5 & 0.4 & 0.2 \\
\hline$\beta$-ocimene-Z & 1035 & 0.5 & 0.0 & 0.0 & 0.0 & 0.0 & 0.0 & 0.0 & 0.0 & 0.1 & 0.1 & 0.0 & 0.1 & 0.1 & 0.1 \\
\hline$\beta$-ocimene-E & 1046 & 0.1 & 0.0 & 0.0 & 0.0 & 0.1 & 0.0 & 0.0 & 0.2 & 0.4 & 0.8 & 0.2 & 1.1 & 0.2 & 0.4 \\
\hline$\delta$-terpinene & 1058 & 1.1 & 0.0 & 0.0 & 0.2 & 0.3 & 0.4 & 0.0 & 1.2 & 2.8 & 2.5 & 0.6 & 0.1 & 0.8 & 1.0 \\
\hline Terpinolene & 1086 & 11.4 & 1.6 & 1.5 & 1.7 & 0.3 & 0.1 & 0.0 & 0.5 & 0.6 & 0.6 & 9.4 & 1.3 & 2.4 & 3.8 \\
\hline Cymenene-para & 1093 & 0.1 & 0.2 & 0.1 & 0.0 & 0.0 & 0.0 & 0.0 & 0.0 & 0.0 & 0.0 & 0.1 & 0.1 & 0.1 & 0.1 \\
\hline Linalool alcool & 1098 & 0.0 & 0.2 & 0.1 & 0.0 & 0.0 & 0.3 & 0.2 & 0.1 & 0.2 & 0.1 & 0.1 & 0.2 & 0.1 & 0.1 \\
\hline Nonanal-n & 1101 & 0.0 & 0.1 & 0.1 & 0.0 & 0.0 & 0.0 & 0.0 & 0.0 & 0.0 & 0.0 & 0.0 & 0.1 & 0.0 & 0.0 \\
\hline Fenchol & 1125 & 0.0 & 0.1 & 0.8 & 0.0 & 0.0 & 0.0 & 0.0 & 0.2 & 0.2 & 0.5 & 0.1 & 0.3 & 0.2 & 0.3 \\
\hline$\alpha$-campholenal & 1133 & 0.0 & 0.0 & 0.9 & 0.0 & 0.0 & 0.1 & 0.1 & 0.0 & 0.0 & 0.0 & 0.0 & 0.0 & 0.1 & 0.3 \\
\hline Sabinol trans & 1148 & 0.0 & 0.1 & 0.0 & 0.0 & 0.0 & 0.2 & 1.1 & 0.0 & 0.0 & 0.0 & 0.0 & 0.0 & 0.1 & 0.3 \\
\hline Pinocarvone & 1172 & 0.0 & 0.0 & 0.0 & 0.0 & 0.0 & 0.0 & 0.1 & 0.0 & 0.0 & 0.0 & 0.0 & 0.0 & 0.0 & 0.0 \\
\hline Isoborneol & 1182 & 0.0 & 0.2 & 0.1 & 0.0 & 0.1 & 0.1 & 0.2 & 0.0 & 0.0 & 0.0 & 0.0 & 0.0 & 0.1 & 0.1 \\
\hline Terpinen-4-ol & 1184 & 0.0 & 0.5 & 0.2 & 0.0 & 0.2 & 0.4 & 0.3 & 0.0 & 0.1 & 0.0 & 0.0 & 0.1 & 0.2 & 0.2 \\
\hline$\alpha$-terpineol & 1186 & 0.0 & 0.1 & 0.1 & 0.3 & 0.0 & 0.0 & 0.0 & 0.2 & 0.4 & 0.2 & 0.2 & 0.4 & 0.2 & 0.2 \\
\hline Cymene 8-ol-para & 1197 & 0.0 & 0.0 & 0.1 & 0.0 & 0.0 & 0.0 & 0.0 & 0.0 & 0.0 & 0.0 & 0.0 & 0.0 & 0.0 & 0.0 \\
\hline Myrtenol & 1206 & 0.0 & 0.5 & 0.0 & 0.0 & 0.0 & 0.1 & 0.0 & 0.0 & 0.0 & 0.0 & 0.0 & 0.0 & 0.0 & 0.1 \\
\hline Isoborneol formate & 1212 & 0.0 & 0.3 & 0.7 & 0.0 & 0.0 & 0.3 & 0.0 & 0.0 & 0.0 & 0.0 & 0.0 & 0.0 & 0.1 & 0.2 \\
\hline Bornyl acetate & 1288 & 0.6 & 1.1 & 0.0 & 1.6 & 1.2 & 0.0 & 1.5 & 0.4 & 1.4 & 0.2 & 0.2 & 0.4 & 0.7 & 0.6 \\
\hline Pinocarvyl acetate & 1297 & 0.2 & 0.0 & 0.0 & 0.0 & 0.0 & 0.0 & 0.0 & 0.0 & 0.1 & 0.0 & 0.0 & 0.0 & 0.0 & 0.1 \\
\hline$\Delta$-elemene & 1354 & 0.0 & 0.4 & 0.6 & 0.7 & 0.3 & 0.8 & 0.6 & 0.2 & 0.6 & 0.2 & 0.2 & 0.3 & 0.4 & 0.3 \\
\hline$\alpha$-ylangene & 1377 & 0.2 & 0.2 & 0.3 & 0.1 & 0.2 & 0.1 & 0.2 & 0.2 & 0.0 & 0.0 & 0.0 & 0.1 & 0.1 & 0.1 \\
\hline$\alpha$-copaene & 1382 & 0.0 & 0.9 & 1.9 & 1.2 & 0.9 & 0.9 & 1.2 & 0.6 & 0.2 & 0.2 & 0.2 & 0.3 & 0.7 & 0.6 \\
\hline$\beta$-bourbonene & 1391 & 0.0 & 0.1 & 0.1 & 0.0 & 0.0 & 0.0 & 0.0 & 0.0 & 0.0 & 0.0 & 0.0 & 0.1 & 0.0 & 0.1 \\
\hline$\beta$-elemene & 1394 & 0.6 & 0.0 & 0.0 & 0.0 & 0.0 & 0.0 & 0.0 & 0.4 & 0.0 & 0.3 & 0.0 & 0.1 & 0.1 & 0.2 \\
\hline Longifolene & 1425 & 0.0 & 0.0 & 0.1 & 0.0 & 0.0 & 0.0 & 0.1 & 0.0 & 0.0 & 0.0 & 0.0 & 0.0 & 0.0 & 0.0 \\
\hline Caryophyllene-Z & 1433 & 2.8 & 17.2 & 1.3 & 7.0 & 3.1 & 10.5 & 0.8 & 4.0 & 3.7 & 0.4 & 1.8 & 1.9 & 4.6 & 4.9 \\
\hline$\delta$-elemene & 1434 & 0.0 & 0.0 & 0.2 & 0.0 & 0.1 & 0.1 & 0.2 & 0.7 & 0.2 & 1.1 & 0.2 & 0.4 & 0.3 & 0.3 \\
\hline Aromadendrene & 1448 & 0.4 & 0.0 & 0.2 & 0.1 & 0.2 & 0.0 & 0.2 & 0.3 & 0.0 & 0.0 & 0.0 & 0.0 & 0.1 & 0.1 \\
\hline$\alpha$-humulene & 1465 & 0.4 & 3.1 & 0.6 & 1.3 & 0.7 & 1.6 & 0.3 & 0.7 & 0.6 & 0.2 & 0.4 & 0.4 & 0.9 & 0.8 \\
\hline Cadina-1 (6)-4- diene-T & 1479 & 0.0 & 0.0 & 0.4 & 0.2 & 0.2 & 0.1 & 0.0 & 0.1 & 0.1 & 0.0 & 0.1 & 0.1 & 0.1 & 0.1 \\
\hline$\delta$-muurolene & 1481 & 0.0 & 2.0 & 2.9 & 1.7 & 1.8 & 1.4 & 2.1 & 0.6 & 0.4 & 0.3 & 0.6 & 0.3 & 1.2 & 0.9 \\
\hline$\alpha$-amorphene & 1485 & 0.0 & 0.5 & 0.5 & 0.4 & 0.3 & 0.3 & 0.3 & 0.1 & 0.0 & 0.0 & 0.1 & 0.1 & 0.2 & 0.2 \\
\hline Germacrene-D & 1489 & 0.2 & 0.3 & 0.4 & 0.5 & 0.2 & 0.7 & 0.2 & 3.1 & 0.0 & 0.1 & 0.3 & 1.2 & 0.6 & 0.8 \\
\hline$\delta$-cadinene & 1498 & 0.1 & 1.0 & 0.2 & 1.0 & 1.2 & 1.0 & 1.3 & 0.0 & 0.2 & 0.0 & 0.2 & 0.2 & 0.5 & 0.5 \\
\hline Muurola-4 (14)5- diene-T & 1501 & 0.2 & 0.1 & 0.9 & 0.1 & 0.0 & 0.0 & 0.0 & 0.6 & 0.2 & 0.1 & 0.2 & 0.2 & 0.2 & 0.3 \\
\hline$\delta$-amorphene & 1502 & 0.0 & 1.2 & 0.9 & 0.8 & 0.9 & 0.6 & 0.7 & 0.0 & 0.0 & 0.0 & 0.0 & 0.0 & 0.4 & 0.5 \\
\hline$\alpha$-muurolene & 1505 & 0.9 & 1.3 & 1.1 & 0.8 & 0.7 & 0.7 & 0.7 & 0.6 & 0.3 & 0.2 & 0.2 & 0.3 & 0.6 & 0.4 \\
\hline$\Delta$-amorphene & 1509 & 0.2 & 0.0 & 0.0 & 0.0 & 0.0 & 0.0 & 0.0 & 0.1 & 0.0 & 0.0 & 0.1 & 0.0 & 0.0 & 0.1 \\
\hline$\Delta$-cadinene & 1520 & 2.5 & 2.2 & 5.3 & 3.2 & 3.2 & 2.4 & 2.5 & 0.3 & 0.7 & 0.3 & 0.8 & 0.6 & 2.0 & 1.5 \\
\hline$\gamma$-bisabolene- E) & 1524 & 0.0 & 0.0 & 0.0 & 0.0 & 0.0 & 0.0 & 0.0 & 1.4 & 0.0 & 0.0 & 0.0 & 0.0 & 0.1 & 0.4 \\
\hline
\end{tabular}




\begin{tabular}{|c|c|c|c|c|c|c|c|c|c|c|c|c|c|c|c|}
\hline Calamenene cis & 1529 & 0.0 & 0.7 & 0.9 & 0.5 & 0.5 & 0.4 & 0.9 & 0.2 & 0.0 & 0.1 & 0.1 & 0.1 & 0.4 & 0.3 \\
\hline Cadina-1,4-D-T & 1539 & 0.3 & 0.3 & 0.5 & 0.3 & 0.3 & 0.3 & 0.1 & 0.2 & 0.2 & 0.1 & 0.1 & 0.1 & 0.2 & 0.1 \\
\hline$\alpha$-cadinene & 1543 & 0.1 & 2.5 & 0.4 & 1.1 & 0.8 & 0.2 & 0.3 & 0.3 & 0.0 & 0.0 & 0.0 & 0.1 & 0.5 & 0.7 \\
\hline Dauca-4 (11),7-diene-T & 1546 & 0.2 & 0.0 & 0.0 & 0.0 & 0.0 & 0.4 & 0.3 & 0.0 & 0.5 & 0.0 & 0.2 & 0.0 & 0.1 & 0.2 \\
\hline$\alpha$-calacorene & 1549 & 0.0 & 2.3 & 0.5 & 1.1 & 0.8 & 0.5 & 0.4 & 0.0 & 0.0 & 0.0 & 0.0 & 0.0 & 0.5 & 0.7 \\
\hline Selina-3,7 (11)-diene & 1552 & 0.0 & 0.0 & 0.0 & 0.0 & 0.0 & 0.0 & 0.0 & 0.3 & 0.6 & 0.0 & 0.2 & 0.1 & 0.1 & 0.2 \\
\hline Germacrene-B & 1570 & 0.0 & 0.6 & 0.2 & 0.2 & 0.0 & 0.3 & 0.1 & 0.6 & 0.2 & 0.8 & 0.2 & 0.3 & 0.3 & 0.3 \\
\hline Hexenyl benzoate-3Z & 1574 & 0.0 & 0.0 & 0.0 & 0.0 & 0.0 & 0.0 & 0.0 & 0.0 & 0.0 & 0.0 & 0.0 & 0.1 & 0.0 & 0.0 \\
\hline Spathulenol & 1587 & 0.0 & 0.0 & 0.0 & 0.0 & 0.0 & 0.0 & 0.0 & 0.2 & 0.0 & 0.0 & 0.0 & 0.1 & 0.0 & 0.1 \\
\hline Caryophyllene oxide & 1593 & 0.0 & 3.9 & 0.6 & 3.5 & 0.7 & 2.0 & 0.4 & 0.4 & 0.3 & 0.1 & 0.1 & 0.2 & 1.0 & 1.3 \\
\hline Globulol & 1596 & 0.0 & 0.0 & 0.0 & 0.0 & 0.0 & 0.0 & 0.0 & 0.1 & 0.0 & 0.0 & 0.0 & 0.0 & 0.0 & 0.0 \\
\hline Salvial 4 (14)en 1-one & 1609 & 0.0 & 0.0 & 0.0 & 0.1 & 0.0 & 0.0 & 0.0 & 0.0 & 0.0 & 0.0 & 0.0 & 0.0 & 0.0 & 0.0 \\
\hline Humulene epoxide II & 1620 & 0.0 & 0.5 & 0.3 & 0.4 & 0.0 & 0.0 & 0.2 & 0.0 & 0.2 & 0.0 & 0.0 & 0.1 & 0.1 & 0.2 \\
\hline$\alpha$-eudesmol-5-7-epi & 1634 & 0.2 & 0.0 & 0.0 & 0.0 & 0.0 & 0.0 & 0.0 & 0.0 & 0.0 & 0.0 & 0.0 & 0.0 & 0.0 & 0.1 \\
\hline Cubenol-1-epi & 1636 & 0.0 & 0.6 & 0.3 & 0.0 & 0.3 & 0.0 & 0.5 & 0.3 & 0.0 & 0.1 & 0.1 & 0.2 & 0.2 & 0.2 \\
\hline$\beta$-muurola 4 (10) (14)dien-ol & 1641 & 0.0 & 0.2 & 0.6 & 1.5 & 0.4 & 0.5 & 0.0 & 0.0 & 0.0 & 0.0 & 0.0 & 0.0 & 0.3 & 0.4 \\
\hline$\delta$-eudesmol & 1642 & 0.0 & 1.2 & 0.0 & 0.3 & 0.2 & 0.3 & 0.0 & 0.3 & 0.4 & 0.3 & 0.2 & 0.2 & 0.3 & 0.3 \\
\hline$\alpha$-muurolol & 1650 & 0.0 & 0.2 & 1.1 & 0.0 & 0.0 & 0.0 & 0.2 & 0.4 & 0.0 & 0.2 & 0.2 & 0.4 & 0.2 & 0.3 \\
\hline Muurolol epi & 1652 & 0.2 & 0.5 & 0.0 & 0.3 & 0.0 & 0.4 & 0.2 & 0.1 & 0.0 & 0.0 & 0.0 & 0.0 & 0.1 & 0.2 \\
\hline Desmethoxyencecalin & 1660 & 0.0 & 0.0 & 0.0 & 0.0 & 0.0 & 0.0 & 0.0 & 0.2 & 0.0 & 0.0 & 0.0 & 1.0 & 0.1 & 0.3 \\
\hline$\alpha$-eudesmol-5-7-epi & 1668 & 0.0 & 4.0 & 0.0 & 1.7 & 1.4 & 1.8 & 0.0 & 1.0 & 2.2 & 0.6 & 0.8 & 0.9 & 1.2 & 1.2 \\
\hline Calamenene 10-ol & 1673 & 0.0 & 0.5 & 0.5 & 0.0 & 0.0 & 0.0 & 0.5 & 0.0 & 0.0 & 0.0 & 0.0 & 0.0 & 0.1 & 0.2 \\
\hline Germacra-4 (15)5,10 (14)-T & 1677 & 0.0 & 0.2 & 0.0 & 0.0 & 0.0 & 0.6 & 0.0 & 0.0 & 0.1 & 0.1 & 0.0 & 0.0 & 0.1 & 0.2 \\
\hline \multicolumn{16}{|l|}{ Chemical classes } \\
\hline Hydrocarbon monoterpenes & & 86.6 & 48.0 & 71.4 & 67.9 & 78.8 & 69.6 & 81 & 79.9 & 85.6 & 93.2 & 91.2 & 87.9 & 78.4 & 12.7 \\
\hline Oxygenated monoterpenes & & 0.8 & 2.9 & 2.9 & 1.8 & 1.6 & 1.5 & 3.3 & 0.9 & 2.4 & 0.9 & 0.6 & 1.4 & 1.8 & 0.9 \\
\hline Hydrocarbon sesquiterpenes & & 9.1 & 36.8 & 20.2 & 22.5 & 16.4 & 23.1 & 13.6 & 15.5 & 8.6 & 4.4 & 6.2 & 7.4 & 15.3 & 9.3 \\
\hline Oxygenated sesquiterpenes & & 0.4 & 11.9 & 3.4 & 7.8 & 3.2 & 5.6 & 1.9 & 2.9 & 3.2 & 1.3 & 1.4 & 2.1 & 3.8 & 3.3 \\
\hline Aldehyde & & 0.0 & 0.1 & 0.1 & 0.0 & 0.0 & 0.0 & 0.0 & 0.0 & 0.0 & 0.0 & 0.0 & 0.1 & 0.0 & 0.1 \\
\hline Ester & & 0.0 & 0.0 & 0.0 & 0.0 & 0.0 & 0.0 & 0.0 & 0.0 & 0.0 & 0.0 & 0.0 & 0.1 & 0.0 & 0.0 \\
\hline Other & & 0.0 & 0.0 & 0.0 & 0.0 & 0.0 & 0.0 & 0.0 & 0.2 & 0.0 & 0.0 & 0.0 & 1.0 & 0.1 & 0.3 \\
\hline
\end{tabular}

Note: $\mathrm{F}=$ female population; $\mathrm{M}=$ male population

With an average of $58.72 \pm 14.45 \%$, the $\alpha$-pinene is the major component in all populations of $R$. tripartita studied; followed by $\Delta 3$-carene $(5.21 \pm 6.89 \%)$, caryophyllene- $Z$ $(4.55 \pm 4.68 \%), \beta$-pinene $(3.07 \pm 1.85 \%)$, myrcene $(2,69 \pm$ $1.96 \%)$, limonene $(2.59 \pm 1.88 \%)$, terpinolene $(2.40 \pm$ $3.64 \%)$ and $\Delta$-cadinene $(2.02 \pm 1.45 \%)$. The terpenes composition of $R$. tripartita essential oils presents certain heterogeneity. The level of $\alpha$-pinene in the populations of Tamanrasset male, M'sila male and Mergueb male is less than $50 \%$, unlike in the other populations the rate varies between 52.02 and $71.88 \%$.

The $\Delta 3$-carene, limonene and terpinolene are present with a significant percentage in Boussaada (male) and Tamanrasset populations. Male populations from M'sila, Maïtar and Mergueb show a high percentage of caryophyllene-Z. The highest percentage of $\Delta 3$-cadinene is observed in the female M'sila population with a rate of $5.33 \%$; the remaining populations contain rates varying between $0.33 \%$ and $3.24 \%$.

The essential oils of Rhus tripartita is rich in hydrocarbon monoterpenes and sesquiterpenes. The hydrocarbon monoterpenes are more abundant in the female populations, however; the male populations contain high levels of hydrocarbon sesquiterpenes.

The hydrocarbon monoterpenes are abundant in all populations with an average of $78.39 \pm 12.65 \%$ (Figure 3), particularly in female populations. On the other hand male population's present high levels of hydrocarbon sesquiterpenes.

The female Chaïba population reveals the highest value of hydrocarbon monoterpenes (93.22\%), on the other hand, the lowest value was found in the samples of the male M'sila population with $47.98 \%$. Hydrocarbon sesquiterpenes are present with an average of $15.32 \pm$ 9.3\%. The aldehydes, esters and other components are present with low values in all the populations studied.

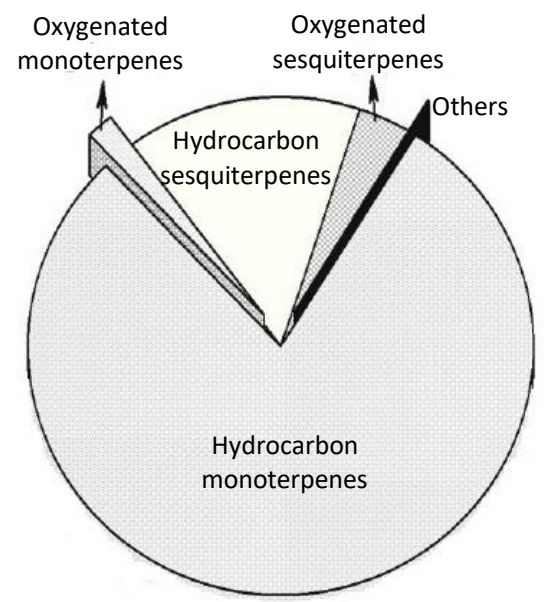

Figure 3. Chemical classes' distribution of essential oils of Rhus tripartita 
The analysis of essential oils shows a difference between the chemical composition of male and female $R$. tripartita populations. The female populations are rich in $\alpha$ pinene $(54.80-78.43 \%)$ and $\beta$-pinene $(1.62-6.40 \%)$, while the male populations show high levels of limonene (0.76$6.03 \%)$, terpinolene $(0.14-9.39 \%)$ and caryophyllene $(\mathrm{Z})$ (1.80-17.19\%). The statistical analysis (Box whisker) of the chemical composition of Rhus tripartita essential oils reveals a significant variability of the components; $\alpha$ pinene with an average $58.72 \pm 14.45 \%$, followed by $\Delta 3$ carene, caryophyllene- $Z$ and terpinolene (Figure 4).

The three-dimensional spatial projection of populations based on the three main axes from the ACP shows that the populations studied are dispersed and remote from each other (Figure 5). They do not gather in homogeneous groups. We notice that certain populations are well individualized like the populations of Tamanrasset and Boussaada male.

The analysis of UPGMA clusters, based on the distance of linkage, indicates the presence of chemical variability between the populations studied. The analysis separated the twelve populations of $R$. tripartita into two main clusters (Figure 6).

The first cluster includes male populations characterized by the presence of low levels of $\alpha$-pinene and high rate of $\Delta 3$-carene, limonene and caryophyllene-Z. It is divided into two groups, Tamanrasset and Boussaada male populations which are characterized by a very high rate of $\Delta 3$-carene (15.30 to $22.3 \%$ ), $\alpha$-pinene (35.84 to $52,02 \%$ ), limonene (6.03-7.23\%) and terpinolene (9.39-11.41\%). The second group is formed by the male populations of M'sila and Mergueb, which are characterized by $\alpha$-pinene $(34.57 \%-43.50 \%), \Delta 3$-carene $(6.24 \%-7.13 \%)$, and caryophyllene-Z (6.99\% - 17.19\%).

The second cluster, rich in $\alpha$-pinene, is subdivided into two groups; the female M'sila population, characterized by $\alpha$-pinene $(54.80 \%), \Delta 3$-carene $(8.34 \%)$ and $\Delta$-cadinene $(5.33 \%)$, represents the first group. The second group, formed mainly by female populations, is characterized by a high level of $\alpha$-pinene (64.40-78.43\%) associated with $\beta$ pinene $(0.65-6.40 \%)$, caryophyllene-Z $(0.37-10.50 \%)$ and myrcene $(0.68-5.34 \%)$.

The chemical analysis and statistical analysis have enabled us to identify four chemotypes in the essential oils of our populations (Table 3 ). The first chemotype with $\Delta 3$ carene $\alpha$-pinene - limonene characterizes the male populations of Tamanrasset and Boussaada. The second chemotype with $\alpha$-pinene - $\Delta 3$-carene - caryophyllene-Z symbolised the male populations of M'sila and Mergueb. The female M'sila population is characterized by the $\alpha$ pinene - $\Delta 3$-carene - $\Delta$-cadinene chemotype. The remaining populations are characterized by the $\alpha$-pinene - $\beta$-pinene caryophyllene- $Z$ chemotype.

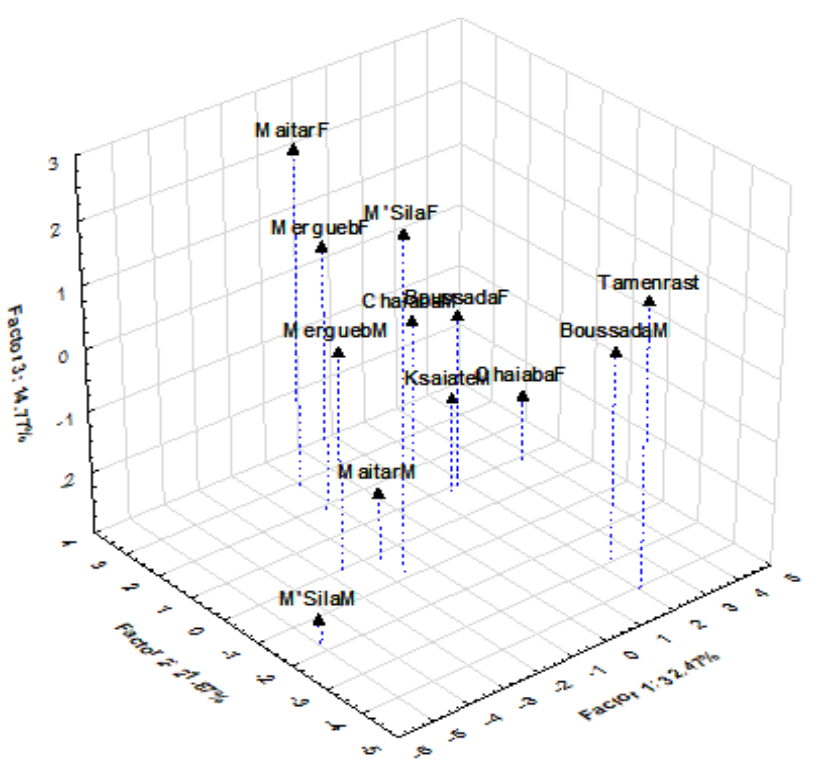

Figure 5. Spatial projection of the Rhus tripartita populations

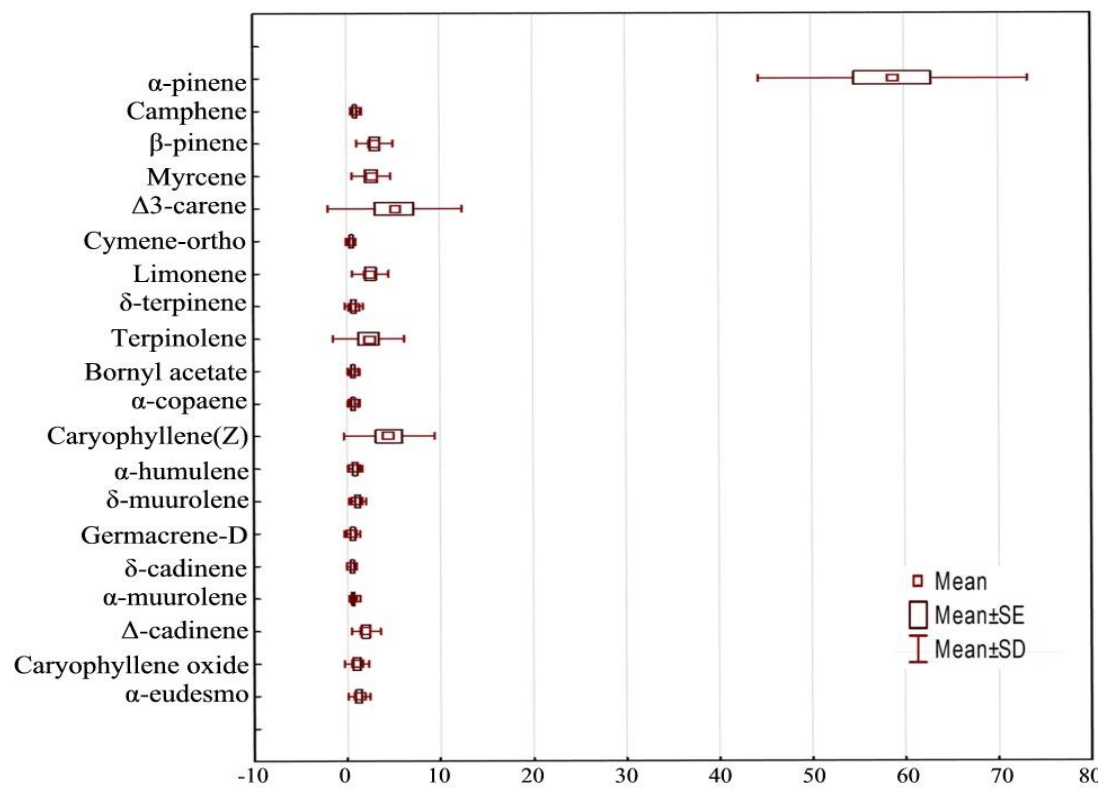

Figure 4. Chemical components variation of Rhus tripartita essential oils 


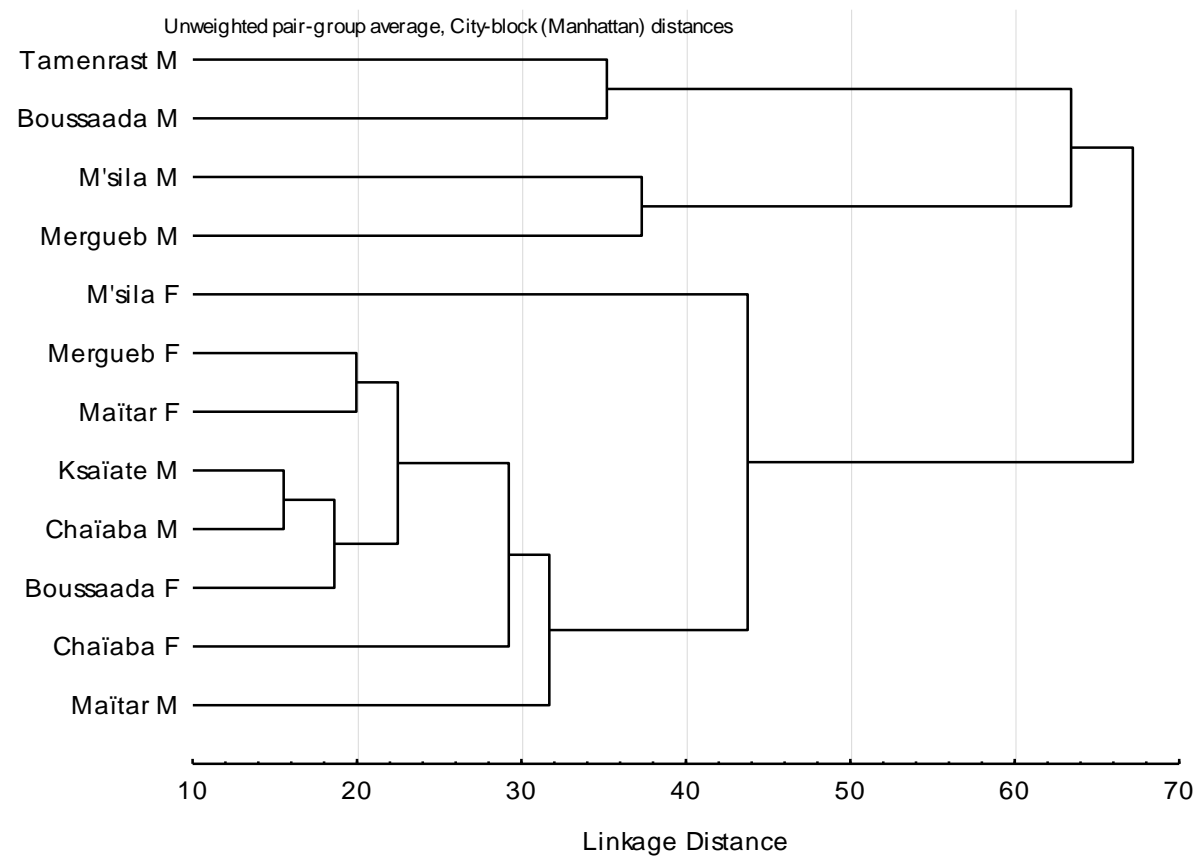

Figure 6. UPGMA cluster of populations of Rhus tripartita in Algeria

Table 3. Chemotypes of Rhus tripartite

\begin{tabular}{|c|c|}
\hline Chemotypes & Populations \\
\hline $\begin{array}{l}\Delta 3 \text {-carene, } \alpha \text {-pinene, } \\
\text { limonene }\end{array}$ & $\begin{array}{l}\text { Tamanrasset and Boussaada } \\
\text { male }\end{array}$ \\
\hline $\begin{array}{l}\alpha \text {-pinene - } \Delta 3 \text {-carene - } \\
\text { caryophyllene-Z }\end{array}$ & M'sila and Mergueb male \\
\hline $\begin{array}{l}\alpha \text {-pinene - } \Delta 3 \text {-carene - } \Delta \text { - } \\
\text { cadinene }\end{array}$ & M'sila female \\
\hline $\begin{array}{l}\alpha \text {-pinene - } \beta \text {-pinene - } \\
\text { caryophyllene-Z }\end{array}$ & $\begin{array}{l}\text { Boussaada female, Mergueb } \\
\text { female, Maïtar (male and } \\
\text { female), Ksaiate (male and } \\
\text { female), Chaïba (male and } \\
\text { female) }\end{array}$ \\
\hline
\end{tabular}

The antibacterial activity of Rhus tripartita essential oils was estimated using the disk diffusion method. After 24 hours of incubation at $37^{\circ} \mathrm{C}$, the antibacterial activity was assessed by measuring the diameters of inhibition zone (Table 4). Antibacterial tests show that $S$. aureus and $E$. coli bacteria are sensitive to the antibiotics used, while $E$. faecalis, $P$. mirabilis and $P$. aeruginosa are resistant to Colistin. The most resistant bacterial strains to the essential oils of $R$. tripartita are $S$. aureus and E. coli. Pure essential oils are the most effective against $E$. faecalis and $P$. aeruginosa. The inhibition of bacterial growth is highly significant; especially in the oil from the Boussaâda male population, whose diameter of inhibition is $55.33 \pm 1.52$ $\mathrm{mm}$.

The essential oils from the populations of Tamenrasset, M'sila, Ksaiate and Chaiba female showed low activity against $P$. mirabilis with an average zone of inhibition of $10.66 \pm 1.5 \mathrm{~mm}$, while the other populations did not affect the growth of this bacteria.
The Rhus tripartita essential oils from M'sila female population exhibits a low antimicrobial activity against the strains tested with an average inhibition diameter of 12.76 $\mathrm{mm}$, although the diameter of inhibition is the largest in the test series. The essential oils from the female population of Mergueb shows the lowest zone of inhibition with a diameter of $11.02 \mathrm{~mm}$ (Table 6).

The antibiotics used in this study were able to inhibit bacterial growth better than $R$. tripartita oils (Table 7). The diameter of inhibition of the essential oils of $R$. tripartita was lower than that of antibiotics with diameters of inhibition inferiors of $4.93 \mathrm{~mm}$. Therefore, these results are not significant in inhibiting the growth of bacteria tested.

The three-way analysis of variance revealed a very significant difference in the diameters of the inhibitory zones among the essential oils. The statistical analysis showed that the Main Effects (Populations, doses, bacterial species, and their interactions are highly significant $(\mathrm{P}<$ 0.001 ) (Table 5).

The bacteria tested are not sensitive to the essential oils of $R$. tripartita (Table 8). The results reveal that $S$. aureus $E$. coli, $P$. mirabilis and $P$. aeruginosa are the most not sensitive to the essential oils of $R$. tripartita. In contrast, $E$. faecalis is the most sensitive to oil.

The desirability profile of Rhus tripartita essential oils against the bacteria tested had a prediction value of 0.246 (Figure 7). The predictive value of essential oils was low. The doses of the oils used in this study have low activity against the bacteria tested, with values not much higher than the predicted value. The desirability test showed that the bacterial species used in this study have values greater than the predicted value, which confirms that these bacteria are resistant to the essential oils of Rhus tripartita. 
Table 4. Inhibition diameter zone ( $\mathrm{mm}$ ) of $R$. tripartita essential oils

\begin{tabular}{|c|c|c|c|c|c|c|c|c|c|c|c|c|c|}
\hline & 苂 & 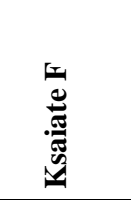 & 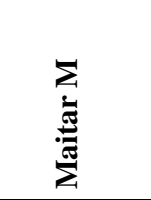 & 吾 & 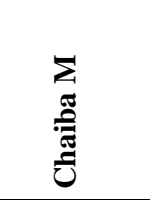 & 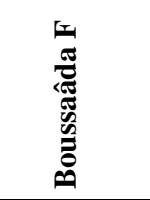 & 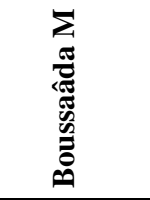 & 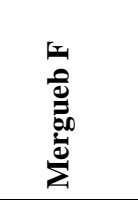 & 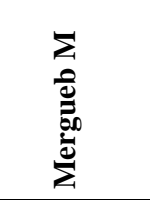 & 至 & $\begin{array}{l}\sum_{j} \\
\frac{\pi}{\bar{c}} \\
\sum\end{array}$ & 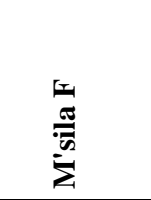 & 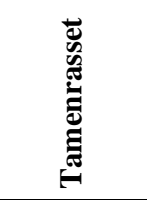 \\
\hline \multirow{8}{*}{ تَّ } & 1 & 0 & 0 & 0 & 0 & 0 & 0 & 0 & 0 & 0 & 0 & 0 & 0 \\
\hline & $1 / 2$ & 0 & 0 & 0 & 0 & 0 & 0 & 0 & 0 & 0 & 0 & 0 & 0 \\
\hline & $1 / 3$ & 0 & 0 & 0 & 0 & 0 & 0 & 0 & 0 & 0 & 0 & 0 & 0 \\
\hline & Colistin & $13 \pm 0$ & $13 \pm 0$ & $13 \pm 0$ & $13 \pm 0$ & $13 \pm 0$ & $13 \pm 0$ & $13 \pm 0$ & $13 \pm 0$ & $13 \pm 0$ & $13 \pm 0$ & $13 \pm 0$ & $13 \pm 0$ \\
\hline & Gentamicin & $22 \pm 0$ & $22 \pm 0$ & $22 \pm 0$ & $22 \pm 0$ & $22 \pm 0$ & $22 \pm 0$ & $22 \pm 0$ & $22 \pm 0$ & $22 \pm 0$ & $22 \pm 0$ & $22 \pm 0$ & $22 \pm 0$ \\
\hline & Cefotaxime & $30 \pm 0$ & $30 \pm 0$ & $30 \pm 0$ & $30 \pm 0$ & $30 \pm 0$ & $30 \pm 0$ & $30 \pm 0$ & $30 \pm 0$ & $30 \pm 0$ & $30 \pm 0$ & $30 \pm 0$ & $30 \pm 0$ \\
\hline & Imipenem & $22 \pm 0$ & $22 \pm 0$ & $22 \pm 0$ & $22 \pm 0$ & $22 \pm 0$ & $22 \pm 0$ & $22 \pm 0$ & $22 \pm 0$ & $22 \pm 0$ & $22 \pm 0$ & $22 \pm 0$ & $22 \pm 0$ \\
\hline & 1 & 0 & 0 & 0 & 0 & 0 & 0 & 0 & 0 & 0 & 0 & $8.33 \pm 0.94$ & 0 \\
\hline \multirow{5}{*}{ 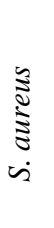 } & $1 / 2$ & 0 & 0 & 0 & 0 & 0 & 0 & 0 & 0 & 0 & 0 & 0 & 0 \\
\hline & $1 / 3$ & 0 & 0 & 0 & 0 & 0 & 0 & 0 & 0 & 0 & 0 & 0 & 0 \\
\hline & Colistin & $20 \pm 0$ & $20 \pm 0$ & $20 \pm 0$ & $20 \pm 0$ & $20 \pm 0$ & $20 \pm 0$ & $20 \pm 0$ & $20 \pm 0$ & $20 \pm 0$ & $20 \pm 0$ & $20 \pm 0$ & $20 \pm 0$ \\
\hline & Gentamicin & $33 \pm 0$ & $33 \pm 0$ & $33 \pm 0$ & $33 \pm 0$ & $33 \pm 0$ & $33 \pm 0$ & $33 \pm 0$ & $33 \pm 0$ & $33 \pm 0$ & $33 \pm 0$ & $33 \pm 0$ & $33 \pm 0$ \\
\hline & Cefotaxime & $30 \pm 0$ & $30 \pm 0$ & $30 \pm 0$ & $30 \pm 0$ & $30 \pm 0$ & $30 \pm 0$ & $30 \pm 0$ & $30 \pm 0$ & $30 \pm 0$ & $30 \pm 0$ & $30 \pm 0$ & $30 \pm 0$ \\
\hline \multirow{8}{*}{ 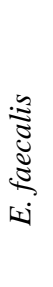 } & Imipenem & $15 \pm 0$ & $15 \pm 0$ & $15 \pm 0$ & $15 \pm 0$ & $15 \pm 0$ & $15 \pm 0$ & $15 \pm 0$ & $15 \pm 0$ & $15 \pm 0$ & $15 \pm 0$ & $15 \pm 0$ & $15 \pm 0$ \\
\hline & 1 & $9.3 \pm 0.6$ & $9 \pm 0$ & $9 \pm 0$ & $25 \pm 4.33$ & $21.66 \pm 1.5$ & $55.33 \pm 1.5$ & $8.66 \pm 0.6$ & $20.33 \pm 1.2$ & $11.33 \pm 2.3$ & $12.66 \pm 0.6$ & $16.66 \pm 3.1$ & $8.33 \pm 1.5$ \\
\hline & $1 / 2$ & $8 \pm 0$ & $6 \pm 4.24$ & $9.33 \pm 0.57$ & $11.33 \pm 4.1$ & $13 \pm 1.73$ & $9.33 \pm 1.52$ & $8 \pm 0$ & $10 \pm 1.73$ & 0 & $9.66 \pm 0.6$ & $5 \pm 3.6$ & 0 \\
\hline & $1 / 3$ & $7.33 \pm 0.6$ & $7.83 \pm 1.04$ & $7.33 \pm 0.6$ & $8.33 \pm 1.2$ & 0 & $5.66 \pm 4.02$ & 0 & 0 & 0 & 0 & 0 & 0 \\
\hline & Colistin & 0 & 0 & 0 & 0 & 0 & 0 & 0 & 0 & 0 & 0 & 0 & 0 \\
\hline & Gentamicin & $20 \pm 0$ & $20 \pm 0$ & $20 \pm 0$ & $20 \pm 0$ & $20 \pm 0$ & $20 \pm 0$ & $20 \pm 0$ & $20 \pm 0$ & $20 \pm 0$ & $20 \pm 0$ & $20 \pm 0$ & $20 \pm 0$ \\
\hline & Cefotaxime & $13 \pm 0$ & $13 \pm 0$ & $13 \pm 0$ & $13 \pm 0$ & $13 \pm 0$ & $13 \pm 0$ & $13 \pm 0$ & $13 \pm 0$ & $13 \pm 0$ & $13 \pm 0$ & $13 \pm 0$ & $13 \pm 0$ \\
\hline & Imipenem & $13 \pm 0$ & $13 \pm 0$ & $13 \pm 0$ & $13 \pm 0$ & $13 \pm 0$ & $13 \pm 0$ & $13 \pm 0$ & $13 \pm 0$ & $13 \pm 0$ & $13 \pm 0$ & $13 \pm 0$ & $13 \pm 0$ \\
\hline \multirow{7}{*}{ 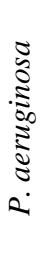 } & 1 & 0 & 0 & $11.33 \pm 0.6$ & 0 & 0 & 0 & 0 & $8.33 \pm 0.6$ & $10 \pm 0$ & 0 & $18 \pm 1.7$ & $11.33 \pm 0.6$ \\
\hline & $1 / 2$ & $9.16 \pm 0.2$ & 0 & $9 \pm 1$ & 0 & 0 & 0 & 0 & 0 & 0 & 0 & $13.66 \pm 2.1$ & $9.33 \pm 0.6$ \\
\hline & $1 / 3$ & 0 & 0 & $8.66 \pm 1.52$ & 0 & 0 & 0 & 0 & 0 & 0 & 0 & 0 & $8.66 \pm 0.6$ \\
\hline & Colistin & $14 \pm 0$ & $14 \pm 0$ & $14 \pm 0$ & $14 \pm 0$ & $14 \pm 0$ & $14 \pm 0$ & $14 \pm 0$ & $14 \pm 0$ & $14 \pm 0$ & $14 \pm 0$ & $14 \pm 0$ & $14 \pm 0$ \\
\hline & Gentamicin & $26 \pm 0$ & $26 \pm 0$ & $26 \pm 0$ & $26 \pm 0$ & $26 \pm 0$ & $26 \pm 0$ & $26 \pm 0$ & $26 \pm 0$ & $26 \pm 0$ & $26 \pm 0$ & $26 \pm 0$ & $26 \pm 0$ \\
\hline & Cefotaxime & $26 \pm 0$ & $26 \pm 0$ & $26 \pm 0$ & $26 \pm 0$ & $26 \pm 0$ & $26 \pm 0$ & $26 \pm 0$ & $26 \pm 0$ & $26 \pm 0$ & $26 \pm 0$ & $26 \pm 0$ & $26 \pm 0$ \\
\hline & Imipenem & 0 & 0 & 0 & 0 & 0 & 0 & 0 & 0 & 0 & 0 & 0 & 0 \\
\hline \multirow{7}{*}{ 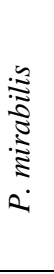 } & 1 & 0 & 0 & $7.33 \pm 0.6$ & 0 & 0 & 0 & 0 & 0 & 0 & 0 & 0 & $9.33 \pm 0.6$ \\
\hline & $1 / 2$ & 0 & 0 & $2.33 \pm 4.1$ & 0 & 0 & 0 & 0 & 0 & 0 & 0 & $10.66 \pm 1.5$ & $7.66 \pm 1.2$ \\
\hline & $1 / 3$ & $7.66 \pm 0.6$ & 0 & $2.33 \pm 4.1$ & 0 & 0 & 0 & 0 & 0 & 0 & 0 & $8.33 \pm 1.52$ & $8.33 \pm 0.6$ \\
\hline & Colistin & 0 & 0 & 0 & 0 & 0 & 0 & 0 & 0 & 0 & 0 & 0 & 0 \\
\hline & Gentamicin & 30 & 30 & 30 & 30 & 30 & 30 & 30 & 30 & 30 & 30 & 30 & 30 \\
\hline & Cefotaxime & 31 & 31 & 31 & 31 & 31 & 31 & 31 & 31 & 31 & 31 & 31 & 31 \\
\hline & Imipenem & 11 & 11 & 11 & 11 & 11 & 11 & 11 & 11 & 11 & 11 & 11 & 11 \\
\hline
\end{tabular}


Table 5. Main effects and interactions of Rhus tripartita essential oils

\begin{tabular}{lllll}
\hline Sources & df & MS & F & P \\
\hline Main effects & & & & \\
$\quad$ Populations & 11 & 41.49 & 97.45 & $.0000 * * *$ \\
$\quad$ Bacteria & 4 & 612.41 & 1438.43 & $.0000 * * *$ \\
$\quad \begin{array}{llll}\text { Doses } \\
\text { Interaction }\end{array}$ & 6 & 20744.71 & 48725.15 & $.0000 * * *$ \\
$\quad$ Populations* Bacteria & 44 & 56.15 & 131.90 & $.0000 * * *$ \\
$\quad$ Populations * Doses & 66 & 24.91 & 58.52 & $.0000 * * *$ \\
$\quad$ Bacteria * Doses & 24 & 1719.57 & 4038.94 & $.0000 * * *$ \\
Populations* Bacteria * Doses & 264 & 28.93 & 67.96 & $.0000 * * *$ \\
\hline
\end{tabular}

Table 6 The effectiveness of Rhus tripartita essential oils from several sampling locations

\begin{tabular}{|c|c|c|c|c|c|c|c|c|}
\hline Rank & Populations & Mean & $\mathbf{n}$ & \multicolumn{5}{|c|}{$\begin{array}{c}\text { Non-significant } \\
\text { ranges }\end{array}$} \\
\hline 1 & M'sila F & 12.76 & 105 & $\mathrm{a}$ & & & & \\
\hline 2 & Boussaâda M & 12.47 & 105 & & $\mathrm{~b}$ & & & \\
\hline 3 & Chaiba F & 12.45 & 105 & & $\mathrm{~b}$ & & & \\
\hline 4 & Tamenrasset & 12.26 & 105 & & & $\mathrm{c}$ & & \\
\hline 5 & Chaiba M & 11.82 & 105 & & & d & & \\
\hline 6 & Mergueb M & 11.81 & 105 & & & $\mathrm{~d}$ & & \\
\hline 7 & Ksaiate F & 11.73 & 105 & & & $\mathrm{~d}$ & & \\
\hline 8 & Boussaâda F & 1145 & 105 & & & & $\mathrm{e}$ & \\
\hline 9 & Maitar M & 11.20 & 105 & & & & & $\mathrm{f}$ \\
\hline 10 & M'sila M & 11.10 & 105 & & & & & $\mathrm{f}$ \\
\hline 11 & Maitar F & 11.07 & 105 & & & & & $\mathrm{f}$ \\
\hline 12 & Mergueb F & 11.02 & 105 & & & & & $\mathrm{f}$ \\
\hline
\end{tabular}

Note: LSD $0.05=0.1767$
Table 7. Effect of oil dilutions on the inhibitory zone of bacteria tested

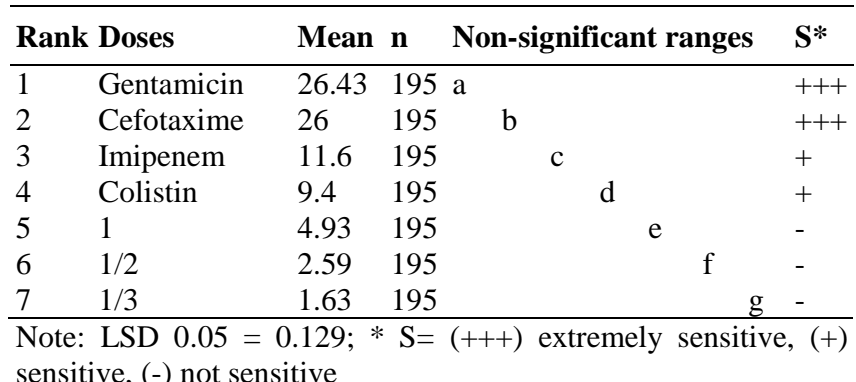

Table 8. Effect of oil dilutions on the inhibitory zone of bacteria tested

\begin{tabular}{lllllllll}
\hline Rank Bacteria & Mean & n & \multicolumn{2}{c}{$\begin{array}{c}\text { Non-significant } \\
\text { ranges }\end{array}$} \\
\hline 1 & S. aureus & 14.09 & 273 & $\mathrm{a}$ & & & \\
2 & E. coli & 12.43 & 273 & & $\mathrm{~b}$ & & & \\
3 & P. mirabilis & 11.36 & 273 & & & $\mathrm{c}$ & & \\
4 & P. aeruginosa & 10.04 & 273 & & & $\mathrm{~d}$ & \\
5 & E. faecalis & 10.06 & 273 & & & & $\mathrm{e}$ \\
\hline
\end{tabular}
sensitive, (-) not sensitive 


\section{Discussion}

The essential oils yield of the Rhus tripartita populations in Algeria is low $(0.16 \%)$. This yield represents some similarity with the yield of $R$. coriaria, which varies between 0.04 and $0.19 \%$ (Morshedlooa et al. 2017). The essential oils yield variation is influenced by several environmental factors, such as drought and salinity (Aghaei et al. 2013; Sangwan et al. 2001); as well as the harvest after flowering, where $70 \%$ of essential oils are evaporated in the air (Salle and Pelletier 1991).

The results of the chemical composition of the essential oils of $R$. tripartita in Algeria show a certain resemblance to other Rhus species, especially in the major components, $\alpha$-pinene, $\Delta 3$-carene, caryophyllene-Z, limonene, and $\Delta 3$ cadinene. Egyptian sumac is rich in cymene-o, $\beta$-ocimene and limonene, while Jordanian and Palestinian Rhus specimens are rich in $\alpha$-pinene and naphthalene (Farag et al. 2018).

The essential oils of $R$. coriaria from Turkey are characterized by $\alpha$-pinene, limonene, nonanol, $\beta$ caryophyllene, $\alpha$-terpineol and carvacrol (Kurucu et al. 1993). The chemical composition analysis of essential oils of $R$. coriaria by Brunke et al. (1993) in Turkey, showed the dominance of hydrocarbon monoterpenes ( $\alpha$-pinene and limonene), followed by hydrocarbon sesquiterpenes ( $\beta$ caryophyllene) and oxygenated sesquiterpenes (caryophyllene oxide and $\beta$-caryophyllene alcohol).

The analysis of flowers and leaves oils of Rhus coriaria of Italy showed that hydrocarbon sesquiterpenes are dominant in leaves, while hydrocarbon monoterpenes are major constituents of the flowers (Reidel et al. 2017), on the other hand, the essential oils of Rhus coriaria fruit in Sicily presented an abundance of non-terpene compounds (Giovanelli et al. 2017). Zhu et al. (2007) made the same findings on the chemical composition of Rhus typhina leaves in China. This variation in chemical composition is caused by variations in habitat, climatic conditions, as well as sampling conditions and genetic factors (Morshedlooa et al. 2017). The richness of $R$. tripartita in hydrocarbon monoterpenes, as well as aromatic monoterpenes such as limonene, $\gamma$-terpinene, and terpinolene explains the characteristic therapeutic properties of this species (Deschepper 2017; Thormar 2011).

The chemotypes of $R$. tripartita, identified in our study, are based on two major components ( $\alpha$-pinene and $\Delta 3$ carene), followed by limonene and caryophyllene-Z, while the chemotypes identified in the Iranian populations of $R$ coriaria are characterized by caryophyllene-E, $\alpha$-pinene and cembrene (Morshedlooa et al. 2017). The results of the antibacterial activity showed that the bacteria tested are moderately sensitive to the $R$. tripartita essential oils. The bacteria $E$. faecalis and $P$. aeruginosa are the most sensitive to the oil, while $P$. mirabilis, $S$. aureus and $E$. coli are the most resistant to oils from all populations.

Our results are in agreement with the work of Bereksi et al. (2019), which showed that extracts from the aerial parts of $R$. tripartita have weak antibacterial activity against $S$. aureus and E. coli. On the other hand, the results of Tlili et al. (2019) confirm that the methanolic extracts of $R$. tripartita have a weak activity on Gram-negative bacteria. Chloroform extracts of $R$. tripartita have significant activity against $S$. aureus and moderate activity against $E$. coli (Abbasi and Hani 2012), while methanolic extracts from the roots, stems and leaves of $R$. tripartita have significant antibacterial activity against $P$. aeruginosa (Ben Barka et al. 2019).

In conclusion, the analysis of Rhus tripartita essential oils by GC/MS, allowed us to identify seventy-eight compounds of which $\alpha$-pinene is the major component, along with $\Delta 3$-carene and caryophyllene-Z. The results reveal a notable difference between the chemical composition of essential oils of male populations and female populations. In this study, four chemotypes were identified in $R$. tripartita. The essential oils of $R$. tripartita shows moderate activity against the bacterial strains tested. $E$. faecalis and $P$. aeruginosa are the most sensitive to oils, while $S$. aureus, E. coli and P. mirabilis are the most resistant.

\section{ACKNOWLEDGEMENTS}

This study was supported financially by MESRS of Algeria (Project code No. D01N01UN190120200005); and no potential conflicts of interest.

\section{REFERENCES}

Abbassi F, Hani K. 2012. In vitro antibacterial and antifungal activities of Rhus tripartitum used as antidiarrheal in Tunisian folk medicine. Nat Prod Res 26 (23): 2215-2218. DOI: 10.1080/14786419.2011.639072.

Abd El-Salam IM. 2015. Phytoconstituents and the study of antioxidant, antimalarial and antimicrobial activities of Rhus tripartita growing in Egypt. J Pharmacogn Phytochem 4 (2): 276-81.

Adams RP. 2007. Identification of Essential Oil Components by Gas Chromatography/Mass Spectrometry. Allured Publishing Corporation, Illinois.

Amin A, Mousa M. 2007. Merits of anti-cancer plants from the Arabian Gulf region. Cancer Ther 5: 55-66.

Ben Barka ZB, Lahbib K, Aouadhi C, Ladjimi MH, Miled HB, Rhouma KB, Sakly M, Maaroufi A, Schneider YJ, Tebourbi O. 2019. Antioxidant, Antimicrobial and Anti-Inflammatory Activities of Edible Rhus tripartita (Ucria) Hydromethanolic Extracts. J Pharm Pharmacol Res 3 (3): 074-087. DOI: 10.26502/jppr.0022

Ben Mahmoud S, Saad H, Charrier B, Pizzi A, Rode K, Ayed N, Charrier B. 2015. Characterization of sumac (Rhus tripartitum) root barks tannin for a potential use in wood adhesives formulation. Wood Sci Technol 49 (1): 205-221. DOI: 10.1007/s00226-014-0686-4

Bereksi MS, Hassaïne H, Bekhechi C, Abdelouahid DE. 2018. Evaluation of antibacterial activity of some medicinal plants extracts commonly used in Algerian Traditional Medicine against some pathogenic bacteria. Pharmacogn J 10 (3): 507-512. DOI: 10.5530/pj.2018.3.83

Bestmann HJ, Classen B, Kobold U, Vostrowsky O, Klingauf F, Steinb U. 1988. Steam volatile constituents from leaves of Rhus typhina. Phytochemistry 27 (1): 85-90. DOI: 10.1016/0031-9422 (88)80595-8

Brunke EJ, Hammerschmidt FJ, Schmaus G, Akgul A. 1993. The essential oil of Rhus coriaria L. fruits. Flavour Fragrance J 8 (4): 209-214. DOI: $10.1002 / f f j .2730080408$.

Chung KT, Wong TY, Huang YW, Lin Y. 2010. Tannins and human health: A review. Crit Rev Food Sci Nutr 38 (6): 421-464. DOI: 10.1080/10408699891274273

Deschepper R. 2017. Variabilité de la composition des huiles essentielles et intérêt de la notion de chimiotype en aromathérapie. [Dissertation]. Sciences pharmaceutiques, Université d'Aix-Marseille, Marseille. [France] 
Farag MA, Fayek NM, Abou Reidah I. 2018. Volatile profiling in Rhus coriaria fruit (sumac) from three different geographical origins and upon roasting as analyzed via solid-phase microextraction. PeerJ 6: e5121. DOI: $10.7717 /$ peerj.5121.

Furth DG. 1985. The Natural History of A Sumac Tree, With An Emphasis on the Entomofauna. (Vol. 46). Archon Books, Hamden.

Giovanellia S, Giusti G, Cioni P, Minissale P, Ciccarelli D, Pistelli L. 2017. Aroma profile and essential oil composition of Rhus coriaria fruits from four Sicilian sites of collection. Industr Crops Prod 97: 166-174. DOI: 10.1080/10412905.2014.882278.

Habibi AA, Zubek SA, Abushhiwa MA, Ahmed MO, El-Khodery SA, Osman HY, Bennour EM. 2015. Antibacterial activity of selected Libyan medicinal plants against Pseudomonas aeruginosa and Escherichia coli. J Pharmacogn Phytochem 3 (6): 197-201.

Hadjadj S, Bayoussef Z, Ould El Hadj-Khelil A, Beggat H, Bouhafs Z, Boukaka Y, Khaldi I, Mimouni S, Sayah F, Tey M. 2015. Ethnobotanical study and phytochemical screening of six medicinal plants used in traditional medicine in the North-eastern Sahara of Algeria (area of Ouargla). J Med Plants Res 8 (41): 1049-1059. DOI: 10.5897/JMPR2015.5932.

Idm'hand E, MsandaF, Cherifi K. 2019. Searsia tripartita (Ucria) Moffett: phytochimie, pharmacologie et usages traditionnels. Phytothérapie: $1-$ 5. DOI: $10.3166 /$ phyto-2019-0167.

Kurucu S, Koyuncu M, Güvenç A, Baser KHC, Özek T. 1993. The essential oils of Rhus coriaria L. (Sumac), J Essential Oil Res 5 (5): 481-486. DOI: 10.1080/10412905.1993.9698267.

Le Floc'h E. 1983. Contribution à une étude ethnobotanique de la flore tunisienne. National Government Publication, Tunisia.

Lee SK, Jung HS, Eo WK, Lee SY, Kim SH, Shim BS. 2010. Rhus verniciflua stokes extract as a potential option for treatment of metastatic renal cell carcinoma: Report of two cases. Annals of Oncology 21 (6): 1383-1385. DOI: 10.1093/annonc/mdq154.

Masada Y. 1976. Analysis of essential oils by Gas Chromatography and Mass Spectrometry. John Wiley \& Sons, New York.

Morshedlooa MR, Maggi F, Neko HT, Aghdam MS. 2017. Sumac (Rhus coriaria L.) fruit: Essential oil variability in Iranian populations. Industr Crops Prod 111: 1-7. DOI: 10.1016/j.indcrop.2017.10.002.

NIST. 2002. Mass Spectral Search Program for the NIST/EPA/NIH Mass Spectral Library, vers. 2.0. fiveash data, USA.

Njoroge PW, Opiyo SA. 2019. Some antibacterial and antifungal compounds from root bark of Rhus natalensis. Amer J Chem 9 (5): 150-158. DOI: 10.5923/j.chemistry.20190905.03.

Ponce AG, Fritz R, Del Valle C, Roura SI. 2003. Antimicrobial activity of essential oils on the native microflora of organic Swiss chard. Lebensmittel-Wissenschaft und Technologie 36 (7): 679-684. DOI: 10.1016/S0023-6438 (03)00088-4.

Quézel P, Santa S. 1963. Nouvelle Flore de l'Algérie et des régions désertiques Méridionales. 2 Vol. C.N.R.S, Paris.
Ridel RVB, Cioni PL, Majo L, Pistelli L. 2017. Evolution of volatile emission in Rhus coriaria organs during different stages of growth and evaluation of the essential oil composition. Chem Biodiv 14 (11): 1-12. DOI: $10.1002 / \mathrm{cbdv} .201700270$.

Saadatian M, Aghaei M, Farahpour M, Balouchi Z. 2013. Chemical composition of lavender (Lavandula officinalis L.) extraction extracted by two solvent concentrations. Global J Med Plant Res 1 (2): 214-217

Salle JL, Pelletier J. 1991. Les huiles essentielles, synthèse d'aromathérapie et introduction à la sympathicothérapie. FrisonRoche, Paris.

Sangwan NS, Farooqi AHA, Shabih F, Sangwan RS. 2001. Regulation of essential oil production in plants. Plant Growth Regul 34: 03-21. DOI: 10.1023/A:1013386921596.

Shahat AA, Alsaid MS, Rafatullah S, Al-Sohaibani MO, Parvez MK, AlDosari MS, Exarchou V, Pieters L. 2016. Treatment with Rhus tripartita extract curtails isoproterenol-elicited cardiotoxicity and oxidative stress in rats. BMC Compl Altern Med 16 (351): 1-11. DOI: 10.1186/s12906-016-1318-3.

Srivastava S, Mallavarapu GR, Rai SK, Singh D, Mishra R, Pandey-Rai S, Kumar S. 2006. Composition of the essential oils of the leaves and flowers of Rhus mysurensis Heyne ex Wight \& Arn growing in the Aravalli mountain range at New Delhi. Flav Fragr J 21: 228-229. DOI: $10.1002 / \mathrm{ffj} .1561$.

Thormar H. 2011. Lipids and Essential oils as Antimicrobial Agents. Wiley, Chichester.

Tlili H, Marino A, Ginestra G, Cacciola F, Mondello L, Miceli N, Taviano MF, Najjaa H Nostro A. 2019. Polyphenolic profile, antibacterial activity and brine shrimp toxicity of leaf extracts from six Tunisian spontaneous species. Nat Prod Res 10 (3): 507-512. DOI: 10.1080/14786419.2019.1616725.

Wang Y, Chan FL, Chen S, Leung LK. 2005. The plant polyphenol butein inhibits testosterone-induced proliferation in breast cancer cells expressing aromatase. Life Sci 77 (1): 39-51. DOI: 10.1016/j.lfs.2004.12.014.

Wu T, Callum JL, Wang S, Liu R, Zhu H, Tsao R. 2013. Evaluation of antioxidant activities and chemical characterisation of staghorn sumac fruit (Rhus hirta L.). Food Chem 138 (2-3): 1333-1340. DOI: 10.1016/j.foodchem.2012.10.086.

Zhaleh M, Sohrabi N, Zangeneh M, Zangeneh A, Moradi R, Zhaleh H. 2018. Chemical composition and antibacterial effects of essential oil of Rhus coriaria fruits in the West of Iran (Kermanshah). J Essential Oil Bearing Plants 21 (2): 493-501. DOI: 10.1080/0972060X.2018.1462739.

Zhu B, Ren Z, Nan P, Jiang M, Zhao J, Zhong Y. 2007. Chemical variation in leaf essential oils of Rhus chinensis from eight locations in Southern and Eastern China. Chem Nat Comp 43 (6): 741-43. DOI: 10.1007/s10600-007-0253-4. 